\title{
Relationship of Transfusion Amounts to Old Age and Survival of Adult Acute Leukemia Patients Who Survived beyond 100
}

\section{Days}

\author{
Jeong-Shi Lin ${ }^{1,2,3}$, Jia-Ching Lin ${ }^{1}$, Ying-Ju Chen ${ }^{1}$, Sheng-Hsuan Chien ${ }^{1,2,3}$, Chun-Yu Liu ${ }^{1,2,3}$ \\ and Tzeon-Jye Chiou ${ }^{1,2,3,4}$
}

${ }^{1}$ Division of Transfusion Medicine, Department of Medicine, Taipei Veterans General Hospital, Taipei, Taiwan

${ }^{2}$ Division of Hematology, Department of Medicine, Taipei Veterans General Hospital, Taipei, Taiwan

${ }^{3}$ National Yang Ming Chiao Tung University, School of Medicine, Taipei, Taiwan

${ }^{4}$ Cancer Center, Taipei Municipal Wanfang Hospital, Taipei, Taiwan

\begin{abstract}
Background: The ageing process puts the elderly at risk of developing anemia and thrombocytopenia with increased risk of transfusions. Patients with increased transfusion requirements may be at increased risk of dying because of the clinical conditions which necessitate transfusion.

Study design and methods: We conducted a nationwide 7-year population-based retrospective study using data retrieved from the Taiwan Longitudinal Health Insurance Database 2005. Analysis of factors influencing transfusion amounts as well as univariate and multivariate analyses of overall survival were performed.

Results: Of the 85 patients randomly selected, the mean age was 49 years (range: 20 to 86 years), and $60 \%$ were male. Fourteen patients had acute lymphoid leukemia (ALL) and 71 had acute myeloid leukemia (AML). Median total number of packed red blood cell (PRBC) requirements (132 vs. 26 units, $\mathrm{P}<0.001)$ and platelets pheresis $(\mathrm{PltPh})$ requirement $(65.5$ vs. 27.0 units, $P<0.001$ ) in old patients ( $\geqq 60$ years) were higher than in younger patients ( $<60$ years). In the univariate analyses of prognostic factors using Cox model, increasing age and AML were unfavorable prognostic factors and the following parameters were not significant: gender, amounts of PRBC and PltPh transfused. In the multivariate analysis, the possible detrimental effect of increasing age and AML could not be demonstrated.

Conclusions: For adult acute leukemia patients who survived beyond 100 days, PRBC and PltPh requirements in patients aged 60 years or older were higher than in younger patients. The effect of the transfusion amounts on length of survival was not significant.
\end{abstract}

Keywords

Transfusion, Acute leukemia, Old age, Prognosis

\section{Introduction}

Anemia and thrombocytopenia are common hematologic problems in the elderly [1,2]. In acute leukemia production of normal blood cells is often decreased due to leukemia involving bone marrow, chemotherapeutic agents, and nutritional deficiency. So symptomatic treatment with transfusions has to be considered. However, the influence of old age on transfusion requirements in acute leukemia has rarely been reported.

Prognosis of acute myeloid leukemia (AML) is influenced both by patient-specific and disease-specific factor. Age is the most prominent patient-specific factor, while chromosomal aberrations are the strongest disease-specific factors [3]. Older patients have a poorer performance status and more comorbidity, and their leukemic cells commonly have unfavourable cytogenetics [4].

In general population, transfusion is independently

*Corresponding author: Jeong-Shi Lin, Division of Transfusion Medicine and Hematology, Department of Medicine, Taipei Veterans General Hospital, No. 201, Sec. 2, Shih-Pai Road, Taipei, 11217, Taiwan, Tel: 886-2-2821-2357

Accepted: December 29, 2021

Published online: December 31, 2021

Citation: Lin JS, Lin JC, Chen YJ, et al. (2021) Relationship of Transfusion Amounts to Old Age and Survival of Adult Acute Leukemia Patients Who Survived beyond 100 Days. Clin Hematol Res 4(1):55-58 
Citation: Lin JS, Lin JC , Chen YJ, et al. (2021) Relationship of Transfusion Amounts to Old Age and Survival of Adult Acute Leukemia Patients Who Survived beyond 100 Days. Clin Hematol Res 4(1):55-58

predictive of mortality and there is a dose-response relationship between the transfusion amounts and a reduction of survival [5]. But the relationship of blood transfusion with overall survival in acute leukemia has not previously been reported.

The purpose of this study was to assess the relationship of transfusion amounts to old age and survival of adult patients with acute leukemia.

\section{Material and Methods}

\section{Data source}

The National Health Insurance (NHI) program in Taiwan is an universal insurance system established in 1995, with coverage of over $99 \%$ of the entire residents in Taiwan. There are approximately 25.68 million individuals in the registry of the Taiwan's National Health Insurance Research Database (NHIRD) in 2005. The Longitudinal Health Insurance Health Insurance Database 2005 (LHID 2005) contains claims data of one-million beneficiaries randomly selected from the Registry of Beneficiaries of NHIRD in 2005. Patients were randomly selected from LHID2005 and conducted a retrospective population-based 7-year (from 2005 to 2011) cohort study.

\section{Study sample}

The International Classification of Disease, Revision 9, Clinical Modification (ICD-9-CM) was used for coding the diagnosis of disease. Diagnosis of acute leukemia was confirmed by the registry for Catastrophic Illness Database. Only people who fulfill the criteria of catastrophic diseases are qualified the catastrophic illness certificates. This study cohort comprised adult patients who had newly diagnosed acute leukemia between 1 January 2005 and 31 December 2010. ICD-9-CM code for acute lymphoid leukemia (ALL) is 2040, and codes for acute myeloid leukemia (AML) are 2050, 2060, 2070 and 2080. Follow-up period was extended to December 2011. For the purpose of analysis of the correlation of transfusion amounts with survival, patients who died within the first 100 days after diagnosis were excluded. To limit the study sample to the adult population, patients aged less than 20 years were also excluded. Blood components were supplied by Taipei blood center. One unit of packed red blood cells (PRBC) is obtained from $250 \mathrm{~mL}$ of whole blood by removing the plasma by centrifugation in Taiwan. One unit of platelets pheresis (PltPh) contains $\geqq 3.0 \times 10^{11}$ platelets.

This study was exempted from full review by the institutional Research Ethic Committee (2014-04-001AE).

\section{Transfusion triggers}

The transfusion policy recommends red cell transfusion to these patients at or below hemoglobin $8 \mathrm{~g} / \mathrm{dL}$ and platelet transfusion at or below $20 \times 10^{9} / \mathrm{L}$ platelet count.

\section{Statistical analysis}

The Mann-Whitney $U$ test was used to compare the transfusion amounts of PRBC or PltPh between groups.

Kaplan-Meier method was used for estimation of overall survival (OS). Cox proportional hazard regression model was used to evaluate the independent effects of covariates including age, gender, leukemia types, and amounts of PRBC or PltPh transfused on OS. The log rank test was also applied for univariate analysis of significant categorical factors. Only factors that were significant at $p<0.20$ in the univariable analyses were included in the multivariable analysis.

A two-side $p$-value less than 0.05 was considered statistically significant. Statistical analyses were conducted using SPSS 20 (IBM Corp. Armonk, NY, USA).

\section{Results}

\section{Patient characteristics}

A total of 85 adult patients with acute leukemia were newly diagnosed in the following years: 10 patients from year 2005, 8 patients from year 2006, 13 patients from year 2007, 15 patients from year 2008, 18 patients from year 2009, 21 patients from year 2010. The mean age was 49 years (range: 20 to 86 years) and $60 \%$ were male. Fourteen patients had ALL and 71 had AML.

\section{Analysis of factors influencing transfusion amounts}

Gender and leukemia type (AML or ALL) did not significantly affect transfusion amounts of PRBC and PltPh. PRBC requirement $(P<0.001)$ and PltPh requirement $(P$ $<0.001$ ) in old patients ( $\geqq 60$ years) were higher than in younger patients $(<60$ years) (Table 1$)$.

\section{Analysis of factors influencing survival}

Univariate analysis using Cox model showed that increasing age and AML were unfavorable prognostic factors, and the following parameters were not significant: gender, amounts of PRBC and PItPh transfused (Table 2).

By the log rank test, AML patients had a significantly shorter median OS compared to ALL patients (median OS 292 vs. 535 days, $P=0.042$ ). Patients with AML were significantly older than patients with ALL $(52.3 \pm 15.0$ vs. $33.1 \pm 13.9, P<$ 0.001). In the multivariate analysis using Cox model, age and leukemia type were not factors determining outcome (Table 2).

\section{Discussion}

In this study, patients were randomly selected from multiple institutes in Taiwan. We analyzed the relationship of transfusion amounts to old age and survival of acute leukemia adult patients who survived beyond 100 days.

The ageing process puts the elderly at risk of developing anemia due to multiple factors (e.g. nutritional deficiencies, inflammatory diseases, renal failure, myelodysplastic syndromes). Old patients usually have higher risk to have significant comorbidities and deterioration of organ function than young patients. Anemia is less tolerable for the elderly [6]. This explained our finding that increased PRBC requirement was observed in old acute leukemia patients who survived beyond 100 days after diagnosis. Dawson, et al. found that 
Citation: Lin JS, Lin JC, Chen YJ, et al. (2021) Relationship of Transfusion Amounts to Old Age and Survival of Adult Acute Leukemia Patients Who Survived beyond 100 Days. Clin Hematol Res 4(1):55-58

Table 1: Transfusion amounts (units) in adult acute leukemia patients who survived beyond 100 days $(n=85)$.

\begin{tabular}{|c|c|c|c|c|c|}
\hline \multirow{2}{*}{ Subgroups } & \multirow{2}{*}{$\mathbf{N}$} & \multicolumn{2}{|c|}{ Packed red blood cell } & \multicolumn{2}{|c|}{ Platelets pheresis } \\
\hline & & Median & 95\% C.I. & Median & 95\% C.I. \\
\hline \multicolumn{6}{|l|}{ Gender } \\
\hline Female & 34 & 32.0 & $43.5-117.0$ & 35.5 & $38.7-115.2$ \\
\hline Male & 51 & 34.0 & $46.9-107.8$ & 29.5 & 41.4-112.4 \\
\hline \multicolumn{6}{|l|}{ Age, years } \\
\hline$<60$ & 59 & 26.0 & $28.3-58.5$ & 27.0 & $30.9-66.8$ \\
\hline$\geqq 60$ & 26 & $132.0^{*}$ & $100.0-216.3$ & $65.5^{*}$ & 69.9-211.4 \\
\hline \multicolumn{6}{|c|}{ Leukemia type } \\
\hline ALL & 14 & 36.5 & $23.4-58.2$ & 28.4 & 17.1-68.2 \\
\hline AML & 71 & 34.0 & $58.9-113.0$ & 35.5 & $53.4-114.0$ \\
\hline
\end{tabular}

${ }^{*} P$ - value $<0.05$ by the Mann-Whitney U test, C.I.: Confidence Interval; AML: Acute Myeloid Leukemia; ALL: Acute Lymphocytic Leukemia, One unit of packed red blood cells is obtained from $250 \mathrm{~mL}$ of whole blood by removing the plasma by centrifugation in Taiwan.

Table 2: Univariate and multivariate analyses for overall survival in adult acute leukemia patients.

\begin{tabular}{|l|l|l|l|l|l|l|}
\hline & \multicolumn{3}{|c|}{ Univariate analysis } & \multicolumn{3}{c|}{ Multivariate analysis } \\
\cline { 2 - 6 } & HR & $95 \%$ Cl & P & HR & 95\% Cl & P \\
\hline Age & 1.018 & $1.004-1.032$ & 0.011 & 1.014 & $0.998-1.030$ \\
\hline Sex & 1.027 & $0.822-1.285$ & 0.813 & & & 0.088 \\
\hline AML vs. ALL & 1.830 & $1.015-3.300$ & 0.045 & 0.722 & $0.369-1.414$ \\
\hline RBC amounts & 1.000 & $0.998-1.003$ & 0.666 & & & 0.342 \\
\hline PltPh amounts & 0.999 & $0.997-1.001$ & 0.542 & & & \\
\hline
\end{tabular}

AML: Acute Myeloid Leukemia; ALL: Acute Lymphocytic Leukemia; PltPh: Platelets Pheresis; HR: Hazard Ratio; C.I.: Confidence Interval, Cox proportional hazard regression model was used for analysis.

the number of blood components used was greatest during induction therapy and there was little variation for patient age, prognostic category or intensity of treatment delivered [7].

Elderly patients are more prone to bleeding and infection complications $[8,9]$. Elderly patients had a greater risk for bleeding at equivalent platelet count [10-12]. So platelet requirements must be higher in elder patients. In this study, $\mathrm{PItPh}$ requirements in old patients were higher than in younger patients.

Survival for acute leukemia patients varies depending on patients' and disease characteristics. Kantarjian, et al. reported the experience in older AML patients (age $\geqq 70$ years) with 8-week mortality of $36 \%$ and OS of 4.6 months after leukemia treatment [13]. Outcome in AML worsens with age, at least in part because of higher treatment-related mortality in older patient [14].

Hahn, et al. reported that increasing age was associated with a progressive rise in early mortality rate; and early death (within two months after diagnosis) was observed in $52.7 \%$ of older AML patients [15]. Sorensen's found that increasing age is inversely related to survival in patients with acute leukemia [16].

Alibhai, et al. reported that although the prognosis has improved over time among younger adults, it remains poor among those aged $\geq 60$ years [17]. In this study, our patients were relatively young because more old patients may be excluded by early death. We found that increasing age tends to be correlated with shorter survival, $P=0.088$.

The limitation of this study is that the number of patients is small and the transfusion amounts were not calculated separately in different stages of therapy.

In summary, in adult acute leukemia patients who survived beyond 100 days, old patients need more PRBC and PltPh units than younger patients. Amounts of PRBC or PltPh transfused were not correlated with survival in adult patients with acute leukemia.

\section{Acknowledgments}

JS Lin designed the study, analyzed the data and wrote this paper. JC Lin acquired the data from the NHIRD by PostgreSQL software. YJ Chen, SH Chien, CY Liu, and TJ Chiou reviewed and revised this paper. This study was supported by a grant from Taiwan Clinical Oncology Research Foundation.

\section{Conflict of Interest}

The authors have no any potential financial and nonfinancial conflict of interest to disclose.

\section{References}

1. Najean Y, Lecompte $T$ (1989) Chronic pure thrombocytopenia in elderly patients. An aspect of the myelodysplastic syndrome. Cancer 64: 2506-2510. 
2. Stauder R, Thein SL (2014) Anemia in the elderly: Clinical implications and new therapeutic concepts. Haematologica 99: 1127-1130.

3. Liersch R, Muller Tidow C, Berdel WE, et al. (2014) Prognostic factors for acute myeloid leukaemia in adults-biological significance and clinical use. Br J Haematol 165: 17-38.

4. Buchner T, Berdel WE, Haferlach C, et al. (2009) Age-related risk profile and chemotherapy dose response in acute myeloid leukemia: A study by the german acute myeloid leukemia cooperative group. J Clin Oncol 27: 61-69.

5. Vamvakas EC, Taswell HF (1994) Long-term survival after blood transfusion. Transfusion 34: 471-477.

6. Beyer I, Compte N, Busuioc A, et al. (2010) Anemia and transfusions in geriatric patients: A time for evaluation. Hematology 15: 116-121.

7. Dawson MA, AveryS, McQuilten ZK, et al. (2007) Blood transfusion requirements for patients undergoing chemotherapy for acute myeloid leukemia how much is enough? Haematologica 92: 996997.

8. Leone G, Mele L, Pulsoni A, et al. (1999) The incidence of secondary leukemias. Haematologica 84: 937-945.

9. Medeiros BC (2014) Management considerations in older patients with AML: A 2014 perspective. Rinsho Ketsueki 55: 1803-1807.
10. Cortelazzo S, Finazzi G, Buelli M, et al. (1991) High risk of severe bleeding in aged patients with chronic idiopathic thrombocytopenic purpura. Blood 77: 31-33.

11. Jick $H$, Slone D, Borda IT, et al. (1968) Efficacy and toxicity of heparin in relation to age and sex. N Engl J Med 279: 284-286.

12. Landefeld CS, Goldman L (1989) Major bleeding in outpatients treated with warfarin: Incidence and prediction by factors known at the start of outpatient therapy. Am J Med 87: 144-152.

13. Kantarjian H, Ravandi F, O'Brien S, et al. (2010). Intensive chemotherapy does not benefit most older patients (age 70 years or older) with acute myeloid leukemia. Blood 116: 44224429.

14. Walter RB, Othus M, Borthakur G, et al. (2011) Prediction of early death after induction therapy for newly diagnosed acute myeloid leukemia with pretreatment risk scores: A novel paradigm for treatment assignment. J Clin Oncol 29: 4417-4423.

15. Hahn A, Giri S, Yaghmour G, et al. (2015) Early mortality in acute myeloid leukemia. Leuk Res 39: 505-509.

16. Sorensen JT, Gerald K, Bodensteiner D, et al. (1993) Effect of age on survival in acute leukemia. 1950-1990. Cancer 72: 1602-1606.

17. Alibhai SM, Leach M, Minden MD, et al. (2009) Outcomes and quality of care in acute myeloid leukemia over 40 years. Cancer 115: 2903-2911. 